\title{
The abscopal effect induced by in situ-irradiated peripheral tumor cells in a murine GL261 brain tumor model
}

\author{
Junko Zenkoh $^{1}$, Ariungerel Gerelchuluun ${ }^{2}$, Yuzi Wang ${ }^{1}$, Yoshihiro Miwa ${ }^{2}$, Tadao Ohno ${ }^{3}$, Koji Tsuboi ${ }^{1}$ \\ ${ }^{1}$ Proton Medical Research Center, ${ }^{2}$ Biomedical Sciences, Faculty of Medicine, University of Tsukuba, 1-1-1 Tennodai, Tsukuba, Ibaraki 305-8575, \\ Japan; ${ }^{3}$ Cell Medicine, Inc. 2-1-6-C-B-1 Sengen, Tsukuba, Ibaraki 305-0047, Japan \\ Contributions: (I) Conception and design: K Tsuboi, J Zenkoh; (II) Administrative support: J Zenkoh; (III) Provision of study materials or patients: Y \\ Miwa; (IV) Collection and assembly of data: J Zenkoh, A Gerelchuluun, Y Wang; (V) Data analysis and interpretation: K Tsuboi, T Ohno, J Zenkoh; \\ (VI) Manuscript writing: All authors; (VII) Final approval of manuscript: All authors. \\ Correspondence to: Koji Tsuboi, MD, PhD. Proton Medical Research Center, Faculty of Medicine, University of Tsukuba, 1-1-1 Tennodai, Tsukuba, \\ Ibaraki 305-8575, Japan. Email: tsuboi-k@md.tsukuba.ac.jp.
}

Background: Localized radiotherapy is considered to act as an adjuvant for systemic anti-tumor immunity. We examined whether in situ-irradiated peripheral tumor cells can evoke an abscopal effect in the brain inhibiting malignant tumor growth.

Methods: Syngeneic albino C57BL/6 mice were inoculated with mouse glioma cells (GL261) transfected with the Kusabira Orange fluorescent gene $(G L 261-m K O)$ for monitoring the tumor growth with in vivo imaging system. GL261-mKO cells were subcutaneously implanted in the thigh and irradiated by X-rays (20 Gy) for in situ vaccination. Ex vivo-irradiated GL261-mKO cells were used as a conventional whole-cell vaccine for comparison. Following these treatments, the brain was challenged with the same GL261-mKO cells, and survival analyses were performed by Kaplan-Meier analysis. In addition, IFN- $\gamma$ release from splenocytes and CD8+ cells infiltration into the brain were analyzed.

Results: Both in situ- and ex vivo-irradiated vaccines significantly prolonged the survival of the mice compared to the control group bearing an intracerebral tumor. Although there was no significant difference in survival between the two vaccination methods, in situ-vaccinated mice with local control by irradiation completely rejected the implanted tumor cells in the brain. In contrast, mice with local failure demonstrated a rapid growth of both subcutaneous and challenged intracerebral tumors. The cured mice demonstrated an accumulation of CD8+ cells surrounding the inoculation site, as well as increased release of IFN- $\gamma$ via an ELISPOT assay.

Conclusions: Our results indicate that the X-ray irradiation to peripheral tumors evoked a protective, tumor-specific immune response in the brain when the peripheral tumors were successfully cured by irradiation.

Keywords: Radiotherapy; brain; vaccine; abscopal effect

Submitted Aug 26, 2016. Accepted for publication Nov 30, 2016.

doi: $10.21037 /$ tcr.2017.01.32

View this article at: http://dx.doi.org/10.21037/tcr.2017.01.32

\section{Introduction}

It has been demonstrated that the localized irradiation of in vivo tumors alone can activate systemic antitumor immunity (1-3). This radiation-induced immune activation is termed "the abscopal effect (4)" and is thought to be initiated via "immunogenic cell death" caused by irradiation $(5,6)$. It has been demonstrated that dying or newly dead tumor cells release damage associated molecular patterns (DAMPs) known as "eat me" or "find me" signals that 
activate dendritic cells to recognize released tumor antigens (5,7-9). The resulting cytotoxic T-lymphocytes (CTLs) then circulate, recognize distant tumor cells, and kill them. Although this effect has been experimentally well established, rarely is the abscopal effect encountered in clinical practice $(10,11)$. However, it remains controversial whether the abscopal effect is organ-independent as in some reported cases, brain metastases have appeared while primary and untreated metastases in other organs resolved $(12,13)$. In particular, there have been no clinical reports in our search of the literature including two recent systematic clinical reviews $(10,11)$, in which brain lesions have exhibited shrinkage or disappearance following radiotherapy to extracranial tumors.

The brain lacks a lymphatic system and is considered an immune-privileged site protected by the blood-brain barrier (BBB) at the normal condition (14), which inhibits the infiltration of circulating lymphocytes into the brain. However, when the BBB is disturbed by tumor formation, circulating lymphocytes may enter the brain through the disturbed sites $(14,15)$. Therefore, it should be clarified whether or not circulating tumor specific lymphocytes act against challenged tumors in the brain.

One of the primary animal models used to examine the anti-tumor immune response in the brain is the orthotopic murine GL261 brain tumor model $(16,17)$, in which ex vivo-irradiated tumor cells have been used for vaccination in various reports (16-18). This whole tumor cell vaccine inactivated by irradiation is assumed to activate cellular immunity by inducing the recognition of released tumor antigens by antigen-presenting cells, followed by the activation of tumor-specific cytotoxic T lymphocytes (CTL) in the lymph nodes. However, no studies have demonstrated if the irradiation of the "in situ" peripheral tumors can evoke an anti-tumor effect in the brain.

Therefore, we designed mouse experiments using the established GL261 brain tumor model to examine whether or not in situ-irradiated peripheral tumor cells can induce a protective, tumor-specific immune response in the brain, and to clarify the role of tumor specific lymphocytes in rejecting implanted tumor cells. This experimental mouse setting presented here is not directly assumed in clinical practice; however, examinations using the protocols for this model should help to understand how or under which conditions the in situ radiation-induced abscopal effect is evoked in the brain. In addition, these results may also apply to enhance the abscopal effect for the prevention of metastatic brain tumors.

\section{Methods}

\section{GL261-mKO cells}

The murine glioma cell line, GL261 $(16,17)$ was provided free of charge by Dr. Masahiro Toda (Department of Neurosurgery, Keio University School of Medicine, Tokyo). GL261 cells were maintained in minimum essential Eagle's medium (MEM; Sigma-Aldrich Japan) supplemented with $10 \%$ heat-inactivated fetal bovine serum (NICHIREI BIOSCIENCES INC., Tokyo, Japan), $100 \mu \mathrm{g} / \mathrm{mL}$ streptomycin and $100 \mathrm{U} / \mathrm{mL}$ penicillin (SigmaAldrich Japan, Tokyo, Japan). To monitor the size of implanted tumors using an in vivo imaging system (IVIS) (Perkin Elmer Inc., Waltham, MA, USA), the cells were stably transfected with a plasmid encoding the gene for monomeric Kusabira Orange protein (pCAG-hu2 mKO) using Lipofectamine LTX Reagent with PLUS ${ }^{\mathrm{TM}}$ Reagent (Thermo Fisher Scientific Inc. Waltham, MA, USA), followed by selection of positive clones with a media containing $800 \mu \mathrm{g} / \mathrm{mL}$ of G418 (Geneticin; Sigma-Aldrich Japan, Tokyo, Japan). Consequently, more than 10 G418resistant clonal cell lines stably expressing Kusabira Orange protein at various levels were identified by flow cytometry (FACSCalibur; Becton, Dickinson and Company Japan, Tokyo, Japan). Clonal cells expressing the maximum level of Kusabira Orange protein were selected and named "GL261-mKO." The cells were cultured in MEM with $10 \%$ FBS and antibiotics but without G418 continuously for three months, and subsequently, the fluorescent stability of GL261-mKO cells was confirmed using flow cytometry. The proliferative capacity and $\mathrm{x}$-ray sensitivities of original GL261 cells and GL261-mKO cells were confirmed to be identical by triplicate growth analyses or standard colony formation assays. For inoculation of mice, GL261-mKO cells were harvested by trypsinization, washed twice with phosphate buffered saline and once with MEM without serum or antibiotics, and then resuspended in MEM without fetal bovine serum or antibiotics at a concentration of $6 \times 10^{5} / 5 \mu \mathrm{L}$ for intracranial inoculation and $1 \times 10^{7} / 100 \mu \mathrm{L}$ for subcutaneous inoculation.

\section{Mouse intracranial and subcutaneous tumor model with GL261-mKO (Figure 1)}

Albino C57BL/6 mice $\left[\mathrm{B} 6(\mathrm{Cg}) \mathrm{Tyr}^{\mathrm{c}-2 \mathrm{~J} J} / \mathrm{J}\right]$ were obtained from the Jackson Laboratory (Sacramento, CA, USA). All animal experimental procedures were conducted in accordance with a protocol approved by the Animal Experimentation 

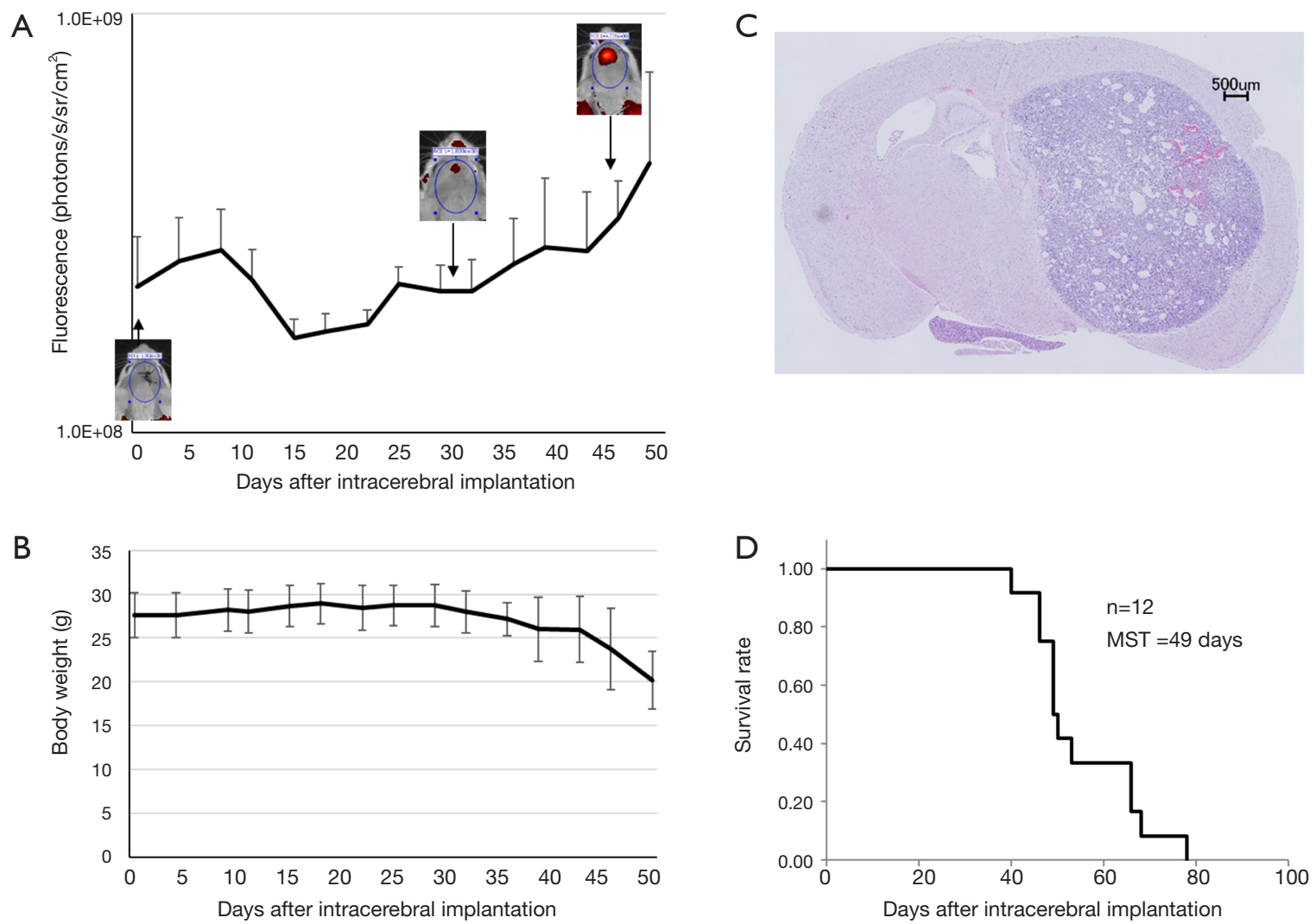

Figure 1 Characteristics of GL261-mKO mouse brain tumor model. (A) Growth curve of implanted the GL261-mKO cells inoculated in to the brain measured by an in vivo imaging system with representative pictures of in vivo imaging. The vertical and the horizontal lines indicate fluorescence intensity and days after inoculation, respectively. The error bars indicate standard deviations; (B) a chronological change of the body weight. The error bars indicate standard deviations; (C) a specimen of a tumor mass 46 days after inoculation, stained by hematoxylineosin; (D) a survival curve of the 12 mice implanted with the GL261-mKO cells into the brain. A median survival time (MST) was 49 days. In this mouse brain tumor model, fluorescence from the intracranial tumor cells was detected approximately 40 days after inoculation, and all mice showed tumor engraftment without failure. As the tumors grew after a temporal decrease, the weight of the mice decreased.

Committee of the Faculty of Medicine, University of Tsukuba. Eight- to 14-week-old male albino C57BL/6 mice were anesthetized by intraperitoneal administration of pentobarbital $(50 \mathrm{mg} / \mathrm{kg})$, and the head was immobilized on a stereotactic operating unit (Narishige Co., Ltd., Tokyo, Japan). The scalp incision was made after preparation with $70 \%$ ethanol, and a burr hole was made using a $26-\mathrm{G}$ needle (TERUMO Corporation, Tokyo, Japan) $1.5 \mathrm{~mm}$ lateral to the midline and $3 \mathrm{~mm}$ anterior to the bregma of the skull. Subsequently, using a $10-\mu \mathrm{L}$ Hamilton syringe (SigmaAldrich Japan, Tokyo, Japan), $6 \times 10^{5}$ GL261-mKO cells in $5 \mu \mathrm{L}$ MEM were injected at a $2.5-\mathrm{mm}$ depth from the surface of the skull. The cells were injected slowly for 2 minutes, and the needle was left in place for 1 minute and slowly withdrawn thereafter. After confirmation of no overflow, the incision was manually sutured. In the subcutaneous model, the mice were anesthetized with isoflurane inhalation, and $1 \times 10^{7}$ GL261-mKO cells in $100 \mu \mathrm{L}$ MEM were then subcutaneously inoculated into the left femoral region using a $1-\mathrm{mL}$ syringe (TERUMO Corporation) attached to a $27 \mathrm{G}$ needle (TERUMO Corporation). In both the intracranial and subcutaneous tumor models, the tumor size was monitored by IVIS every 3 to 7 days for over 110 days following subcutaneous inoculation. Since the white hair of the mice disturbed the Kusabira Orange fluorescence, the tumor sites were depilated before IVIS detection. The mice were euthanized by cervical dislocation when severe neurological symptoms or weight reduction $\geq 20 \%$ appeared; this was in compliance with the "Guideline for Endpoints in Animal Study 
Proposals" of the NIH (19). The intensity of Kusabira Orange fluorescence detected by IVIS was analyzed using Living Image 4.3.1 software (PerkinElmer Inc., Waltham, MA, USA).

\section{Protocols}

\section{Immune protective protocol using in situ irradiated} tumor cells as vaccine

Ten days after the left femoral region of the mice was subcutaneously inoculated with GL261-mKO cells, the tumors were treated with localized radiation (20-Gy X-rays) using an $\mathrm{X}$-ray generator $(130 \mathrm{kV}, 5 \mathrm{~mA}$ with $0.5 \mathrm{~mm} \mathrm{Cu}$ and $0.1 \mathrm{~mm} \mathrm{Al,} \mathrm{MBR-1520R;} \mathrm{Hitachi} \mathrm{Medical} \mathrm{Co.,} \mathrm{Tokyo,}$ Japan) with a custom-made $10-\mathrm{mm}$ width brass collimator to expose only the subcutaneous tumors sites. Twenty-four days after irradiation, the brain was inoculated with GL261$\mathrm{mKO}$ cells. Both intracranial and subcutaneous tumors were monitored by IVIS. The mice were euthanized by cervical dislocation when severe neurological symptoms or weight reduction $\geq 20 \%$ appeared.

\section{Immune protective protocol using ex vivo irradiated tumor cells for a comparison}

GL261-mKO cells were suspended in MEM at a concentration of $1 \times 10^{7} / 100 \mu \mathrm{L}$ and irradiated with $20-\mathrm{Gy}$ $\mathrm{X}$-rays $(130 \mathrm{kV}, 5 \mathrm{~mA}$ with $0.5 \mathrm{~mm} \mathrm{Cu}$ and $0.1 \mathrm{~mm} \mathrm{Al}$, MBR1520R; Hitachi Medical Co., Tokyo, Japan). Within 30 minutes of irradiation, the left femoral region of the mice was subcutaneously inoculated with GL261-mKO cells. Twentyfour days after vaccination, the brain was also inoculated, and the cells were monitored by IVIS. The mice were euthanized by cervical dislocation when severe neurological symptoms or weight reduction $\geq 20 \%$ appeared.

\section{Re-challenge with the same or a different tumor cell type}

After a 110-day observation, the surviving mice without recurrence in the protective in situ vaccination protocol group were re-inoculated with $6 \times 10^{5}$ GL261-mKO cells or $2.5 \times 10^{4} \mathrm{~B} 16 / \mathrm{BL} 6$ cells (a syngeneic mouse melanoma cell line) in the brain. Mice were observed on alternate days until a point at which severe neurological symptoms or weight reduction $\geq 20 \%$ appeared.

\section{Cluster of Differentiation 8 (CD8) + immunohistochemical staining}

For immunohistochemical staining of the brain, tumor- bearing mice were euthanized, and the brain was removed. Immediately, the brain was dissected and mounted in O.C. $T^{\mathrm{TM}}$ compound (Sakura Finetek Japan Co., Ltd., Tokyo, Japan), and frozen gradually from -20 to $-80{ }^{\circ} \mathrm{C}$. The frozen tissue was sliced into $6-\mu \mathrm{m}$-thick sections using a cryostat (CM1850; Leica Biosystems GmbH, Nussloch, Germany) and placed on precoated glass slides (Matsunami glass ind., ltd., Osaka, Japan). After drying for 10 minutes at room temperature, the tissue sections were fixed with acetone for 10 minutes at $-20{ }^{\circ} \mathrm{C}$. The slides were rinsed twice with phosphate-buffered saline (PBS) for 5 minutes each at room temperature, followed by incubation with $1 \%$ hydrogen peroxide in PBS for 5 minutes at room temperature to block endogenous peroxidase activity in the tissues. Following washing with PBS, the sections were blocked using Universal Blocker ${ }^{\mathrm{TM}}$ in Tris-buffered saline (TBS) (Thermo Fisher Scientific Inc. Waltham, USA) for 30 minutes at room temperature. The slides were washed twice in PBS and then incubated with anti-CD8 $\alpha$ (EP1150Y; Novus biologicals llc. Littleton, CO, USA) diluted in Can Get Signal immunoreaction enhancer solution-A (Toyobo Ltd. Osaka, Japan) for 1 hour at $25^{\circ} \mathrm{C}$. Finally, the sections were stained with biotin-streptavidin using the $\mathrm{LSAB}^{{ }} 2$ system-HRP (DAKO Japan llc, Tokyo, Japan) according to the manufacturer's instructions. When the specimens became brown in color, the reaction was stopped by rinsing with PBS. The specimens were stained with hematoxylin and eosin, dehydrated, penetrated, and covered with glass on the slides. Four mice with local control, 3 mice with brain tumors without pretreatment, and 2 mice with local failure were analyzed.

\section{Enzyme-linked immunospot (ELISPOT) assay}

Mice were euthanized by cervical dislocation, and the spleen was excised and ground using sterilized slideglasses. Following treatment with red blood lysis buffer, splenocytes were harvested, and single-cell suspensions were prepared in MEM. Splenocytes $\left(2.5 \times 10^{6}\right)$ were plated onto 96-well ELISPOT plates coated with purified antimouse interferon (IFN) $\gamma$ antibody. The splenocytes were stimulated with or without GL261-mKO lysate. Following incubation at $37{ }^{\circ} \mathrm{C}$ with $5 \% \mathrm{CO}_{2}$ for 24 hours, the ELISPOT assay was performed according to the manufacturer's manual (Mouse IFN- $\gamma$ ELISPOT Set; Becton, Dickinson and Company Japan). The number of spots were counted by eye. The results were derived from triplicates of each experiment. 


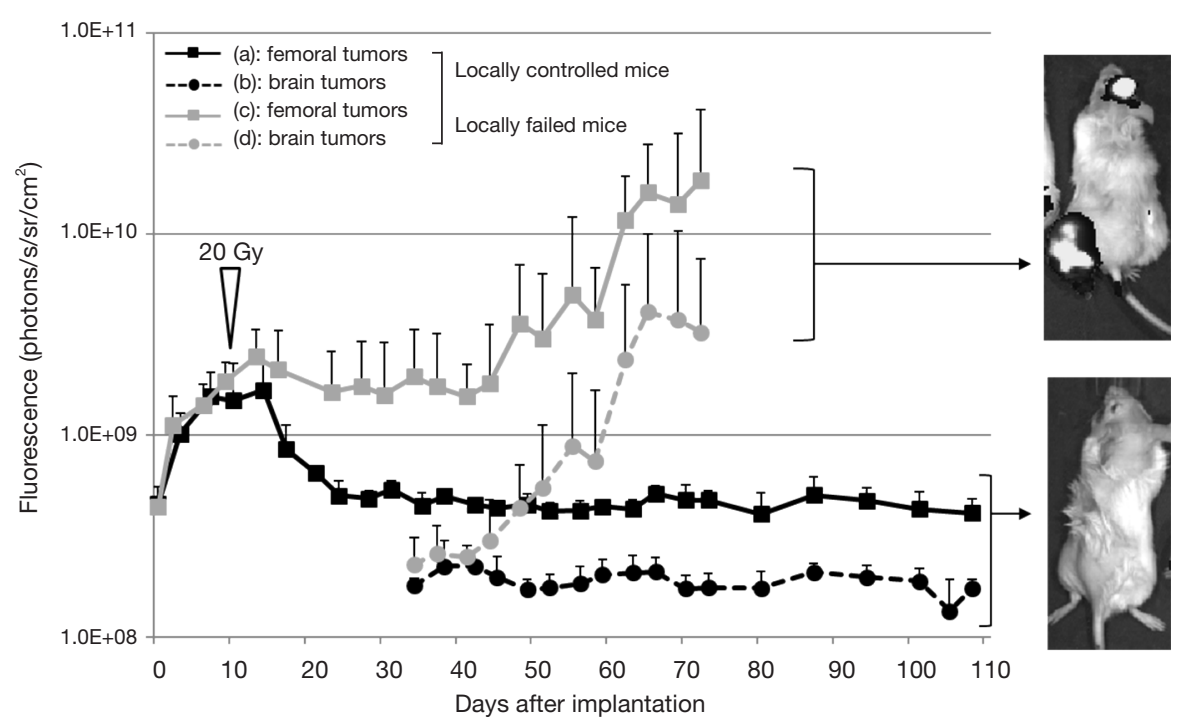

Figure 2 Two distinctly different growth patterns of tumors in the femoral region and in the brain after in situ-vaccination. (a) and (b): the tumors at the femoral region and in the brain in locally-controlled mice $(n=8)$. (c) and $(d)$ : the tumors at the femoral region and in the brain in locally-failed mice $(n=8)$. The error bars indicate standard deviation. Representative pictures of locally-failed and -controlled mice were shown on the right side. In all the 8 mice with cure of subcutaneous tumors (local control), inoculated GL261-mKO cells in the brain were completely rejected, and every mouse survived. In contrast, in all the 8 mice with re-growth of subcutaneous tumors (local failure), GL261mKO cells were engrafted and grew very rapidly in every mouse.

\section{Survival analysis and statistics}

Survival was analyzed using the Kaplan-Meier method, and statistical difference was determined using the log-rank test. A P value less than 0.05 was considered statistically significant.

\section{Results}

\section{GL261-mKO brain tumor mouse model}

The results of the intracranial tumor fluorescence detected by IVIS were shown in Figure 1. Fluorescence from the intracranial tumor cells was detected approximately 40 days after inoculation. All mice showed tumor engraftment without failure, and as the tumors grew after a temporal decrease, the weight of the tumor-bearing mice decreased. The hematoxylin-eosin staining of the brain removed from a tumor-bearing mouse demonstrated a well-circumscribed intracerebral tumor. The median survival time of the 12 mice with intracranial tumors was 49 days (Figure 1).

\section{Effect of in situ-and ex vivo irradiated GL261-mKO cells}

Sixteen mice were subcutaneously inoculated with GL261-
$\mathrm{mKO}$ cells in the left femoral region and were irradiated with 20-Gy X-rays 10 days later. The dose of 20-Gy was selected in this study based on our previous studies in which the skin reaction was within a tolerable range and approximately half of the tumors were cured with this dose. Twenty-four days after irradiation, the brains were inoculated with the same tumor cell type. Among the 16 mice that underwent this protocol, 8 mice showed stabilization and eventual cure of the subcutaneous tumors by day 10 after irradiation. In contrast, in the remaining 8 mice, the subcutaneous tumors grew without shrinkage, or grew after temporal shrinkage followed by rapid growth from 40 to 50 days after X-ray irradiation (Figure 2). In all the 8 mice that were cured of subcutaneous tumors following irradiation (local control), inoculated GL261-mKO cells in the brain were completely rejected, and all the mice survived. In contrast, in all the 8 mice that showed growth of tumors following irradiation (local failure), GL261-mKO cells inoculated in the brain were engrafted and grew very rapidly, and all mice did not survive (Figure 2). In Kaplan-Meier analysis, although the mice treated by the in situ vaccination started to die earlier, the median survival times of the 16 in situ vaccinated mice and 12 control tumor-bearing mice were 63 and 49 days, respectively. There was a significant difference between 

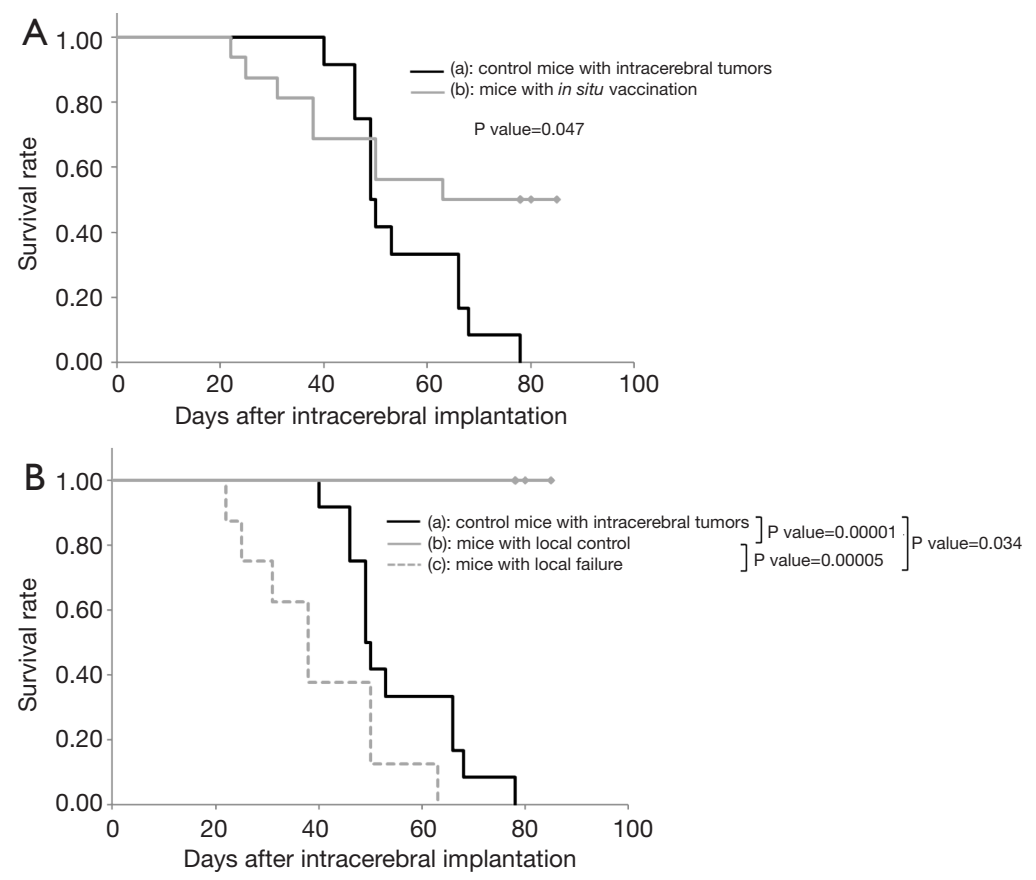

Figure 3 (A) Survival curves of the mice after intracerebral implantation of the GL261-mKO cells. (a): the control mice with intracerebral tumors ( $\mathrm{n}=12, \mathrm{MST}=49$ days). (b): the mice pre-treated by in situ-vaccination by localized irradiation ( $\mathrm{n}=16, \mathrm{MST}=63$ days). There was a significant difference between them; (B) survival curves of the mice after intracerebral implantation of the GL261-mKO cells. (a): the control mice with intracerebral tumors ( $\mathrm{n}=12, \mathrm{MST}=49$ days). (b): the mice pre-treated by in situ vaccination with local control ( $\mathrm{n}=8$, MST: not available). (c): the mice pre-treated by in situ vaccination with local failure ( $\mathrm{n}=8$, MST $=38$ days). There were significant differences between (a) and (b), (a) and (c), and (b) and (c). Although the mice treated by the in situ vaccination started to die earlier, the survival of the mice with in situ vaccination was significantly longer than that of control mice with intracerebral tumors. The survival of the mice treated by in situ vaccination was divided into 2 patterns based on the response of subcutaneous tumor, and the survival of locally-failed mice was significantly worse than that of control tumor-bearing mice.

these two cohorts in the log-rank test with a $\mathrm{P}$ value of 0.047 (Figure $3 A$ ). The 8 mice with local control and the 8 mice with local failure were analyzed separately using the Kaplan-Meier method. There was a significant difference between these two cohorts, with a $\mathrm{P}$ value of less than 0.0001 in the log-rank test (Figure $3 B$ ). In addition, when comparing the 8 locally-failed mice and the 12 tumorbearing control mice, median survival times were 38 and 49 days, respectively, and the survival of locally-failed mice was significantly worse than that of control tumor-bearing mice, with a $\mathrm{P}$ value of 0.034 (Figure 3B).

With respect to $e x$ vivo irradiated whole tumor cell vaccine, GL261-mKO cells were irradiated ex vivo with 20-Gy X-rays, and were subcutaneously inoculated at the left femoral region of 7 mice at 15-30 minutes after irradiation. There was no tumor formation observed at the inoculation site. Among these 7 mice, 3 mice rejected the same tumor cells that had been inoculated in the brain 24 days after vaccination. The remaining 4 mice showed tumor cell engraftment in the brain on day 8 to 29. Consequently, the median survival time of the 7 vaccinated mice was 66 days. Kaplan-Meier analysis demonstrates significant prolongation of survival in this cohort with a $\mathrm{P}$ value of 0.038 compared with the 12 mice bearing intracranial tumors (Figure $4 A$ ). When comparing the survivals of mice treated with in situand ex vivo-irradiated vaccines, although the mice treated by the in situ vaccination started to die earlier, the final survivals were almost the same $(\mathrm{P}=0.927)$ (Figure $4 B)$.

\section{Long-term survivors show a protective response to tumor re-challenge}

To determine whether the long-term surviving mice acquired immunological memory following the protective 

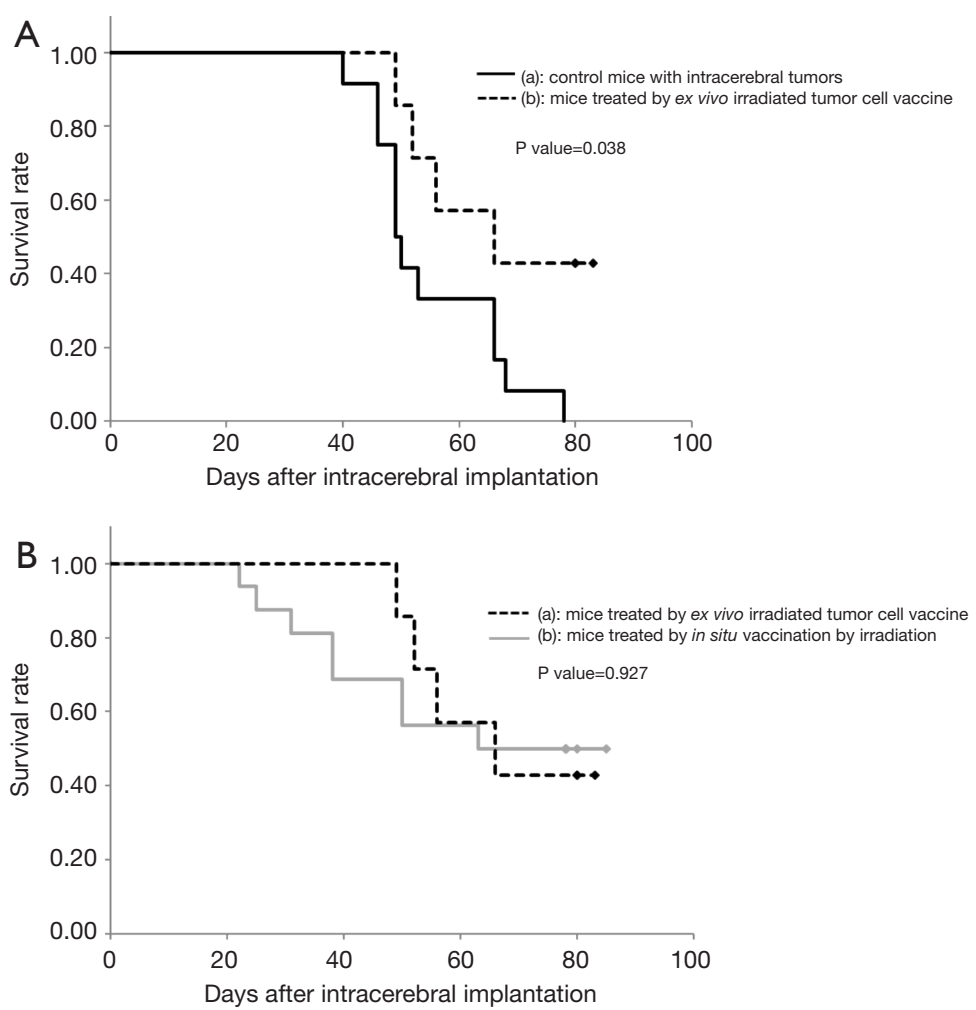

Figure 4 (A) Survival curves of the mice after intracerebral implantation of the GL261-mKO cells. (a): the control mice with intracerebral tumors ( $\mathrm{n}=12, \mathrm{MST}=49$ days). (b): the mice pre-treated by ex vivo irradiated tumor cell vaccine ( $\mathrm{n}=7, \mathrm{MST}=66$ days); (B) survival curves of the mice after intracerebral implantation with the GL261-mKO cells. (A) The mice pre-treated by ex vivo irradiated tumor cell vaccine ( $\mathrm{n}=7$, MST =66 days); (B) the mice pre-treated by in situ vaccination by localized irradiation ( $\mathrm{n}=16$, MST =63 days). There was no significant difference between them. The survival of the mice pre-treated by ex vivo irradiated tumor cell vaccine was significantly longer than that of the control mice bearing intracranial tumors $(\mathrm{P}=0.038)$. Comparing the survivals of mice treated with in situ- and $e x$ vivo- irradiated vaccines, although the mice treated by the in situ vaccination started to die earlier, the final survivals were almost the same $(\mathrm{P}=0.927)$.

protocol, the brains were re-inoculated with GL261-mKO or B16/BL6 cells 91-175 days after the former tumor implantation in the brain. With respect to the analysis of survival, mice that were re-inoculated in the brain with GL261-mKO cells survived without engraftment for over 200 days. In contrast, all the mice that were inoculated with B16/BL6 cells showed neurological symptoms within two weeks of inoculation and were mortal. Kaplan-Meier analysis shows a significant difference between these two groups, with a $\mathrm{P}$ value of 0.0005 (Figure 5).

\section{Infiltration of CD8+CTLs}

Using 4 mice that showed local control of the femoral tumor following irradiation, the brains were removed 7-14 days after intracerebral tumor inoculation. For comparison, brains of 2 locally-failed mice and 3 mice bearing tumor without pretreatment were removed 22-43 days after intracerebral tumor inoculation. Frozen sections were then immunohistochemically stained with an anti-CD8 antibody. In locally-controlled mice CD8+ cells were observed to be accumulated along the track of the needle and around the remaining intracerebral tumor cells (Figure 6). In contrast, CD8+ cells were scarcely observed in the locally-failed mice and the mice bearing brain tumor without pre-treatment (Figure 7).

\section{IFN-y release from splenocytes}

ELISPOT assay was performed to detect the IFN- $\gamma$ released from splenocytes of the 9 treated mice: 4 mice with local control of the femoral tumor, 2 mice with local failure, 


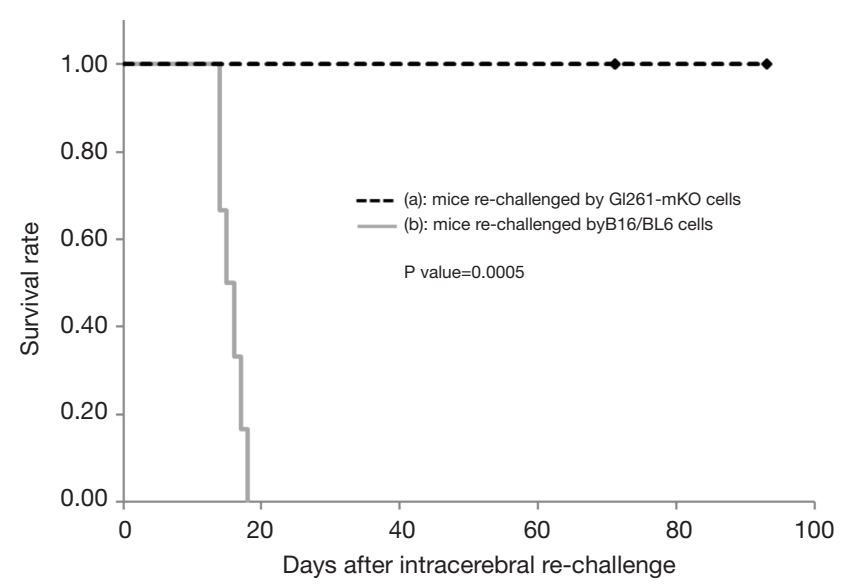

Figure 5 Survival curves of the mice after re-challenged by (A) GL261-mKO cells ( $\mathrm{n}=6$, MST: not available); and (B) B16/BL6 cells ( $\mathrm{n}=6, \mathrm{MST}=15$ days). There was a significant difference between them $(\mathrm{P}=0.0005)$.

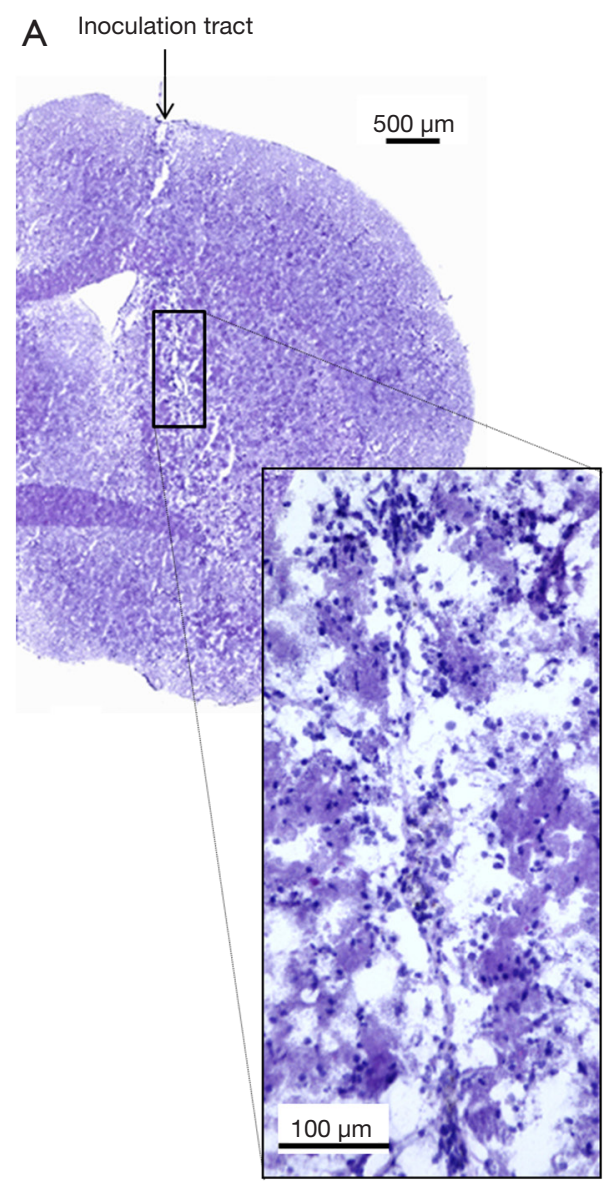

and 3 mice with intracerebral tumor without any pretreatment. In every mouse with local control of the femoral tumor, the number of spots was significantly increased by antigen stimulation. Also, the numbers of spots after antigen stimulation in these mice were significantly larger than those in the mice with local failure $(\mathrm{P}=0.0002)$ and the control mice bearing intracerebral tumors $(\mathrm{P}=0.002)$ (Figure 8). In particular, the numbers of spots were significantly smaller and were not increased by antigen stimulation in the 2 mice with local failure, indicating that the anti-tumor immunity was almost completely suppressed in these mice.

\section{Discussion}

\section{GL261-mKO cells and the mouse brain tumor model}

Murine GL261 cells have been used for a standard

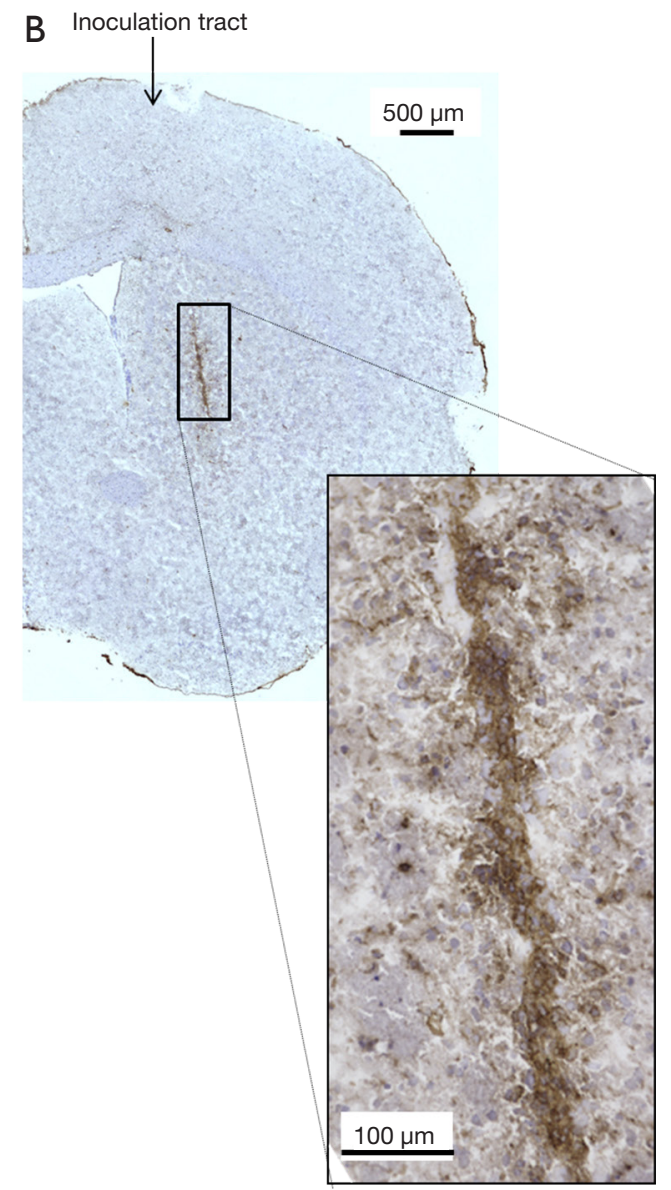

Figure 6 Histological analyses of brain specimens of the mice pre-treated by in situ-vaccination with local control. (A) Hematoxylin-eosin staining; (B) immunohistochemical staining with anti-CD8 antibody with low and high magnification. 
A Inoculation tract

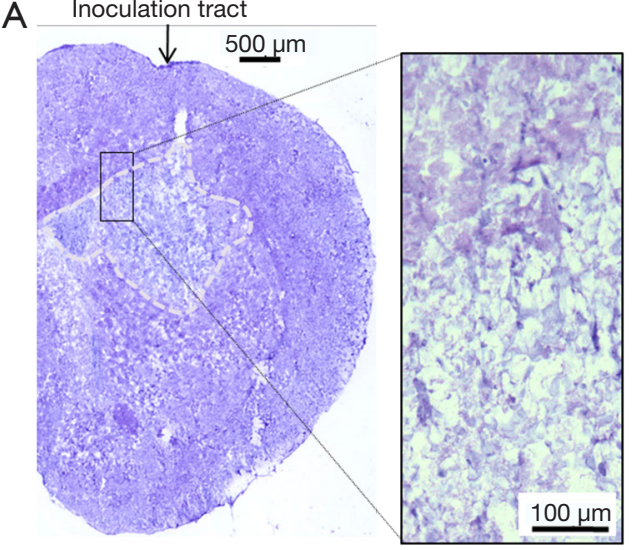

C

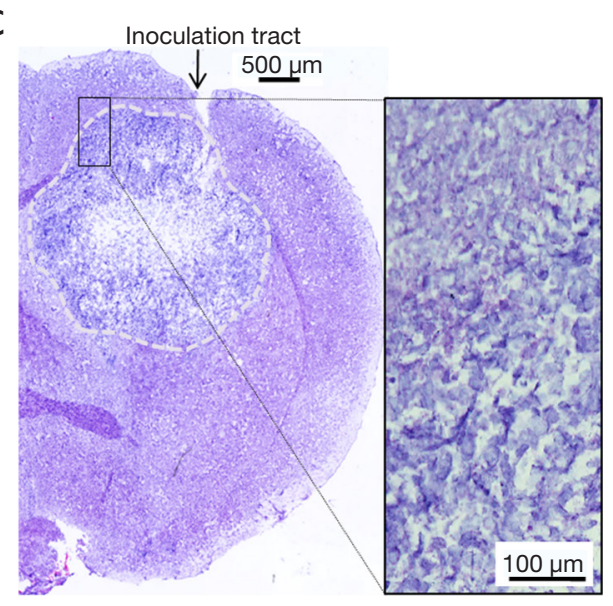

B

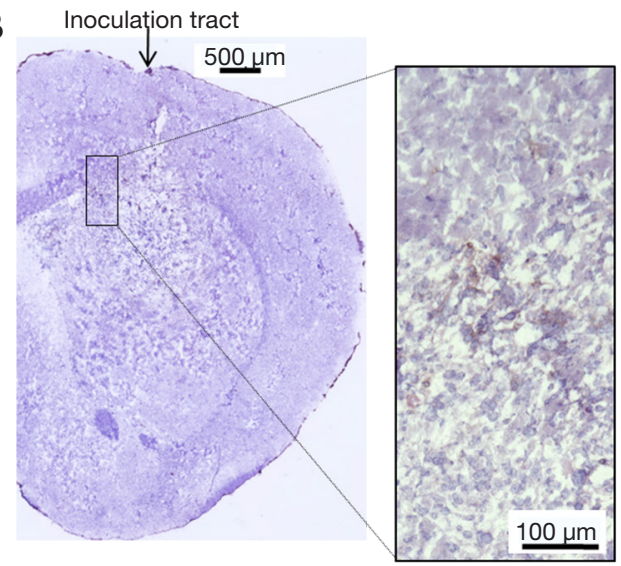

D

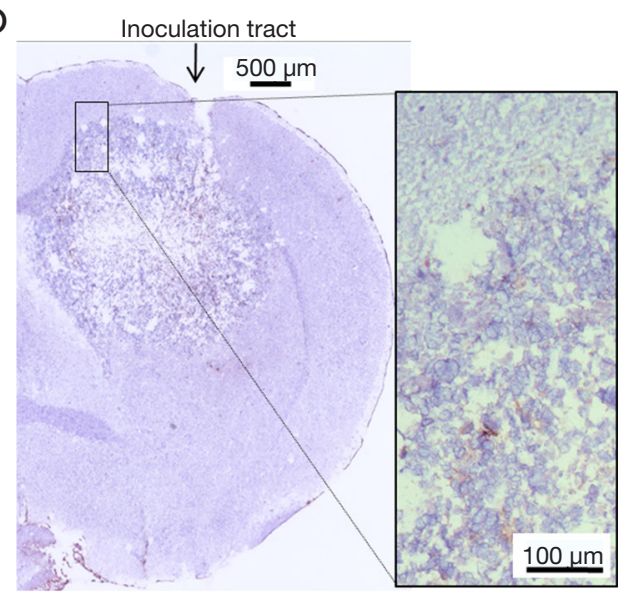

Figure 7 Histological analyses of brain specimens of the mice with intracerebral tumors without pre-treatment $(\mathrm{A}, \mathrm{B})$ and the mice with local failure after in situ-vaccination (C,D); (A,C) hematoxylin-eosin staining. The dotted lines indicate boundaries of the tumor; (B,D) immunohistochemical staining with anti-CD8 antibody with low and high magnification.

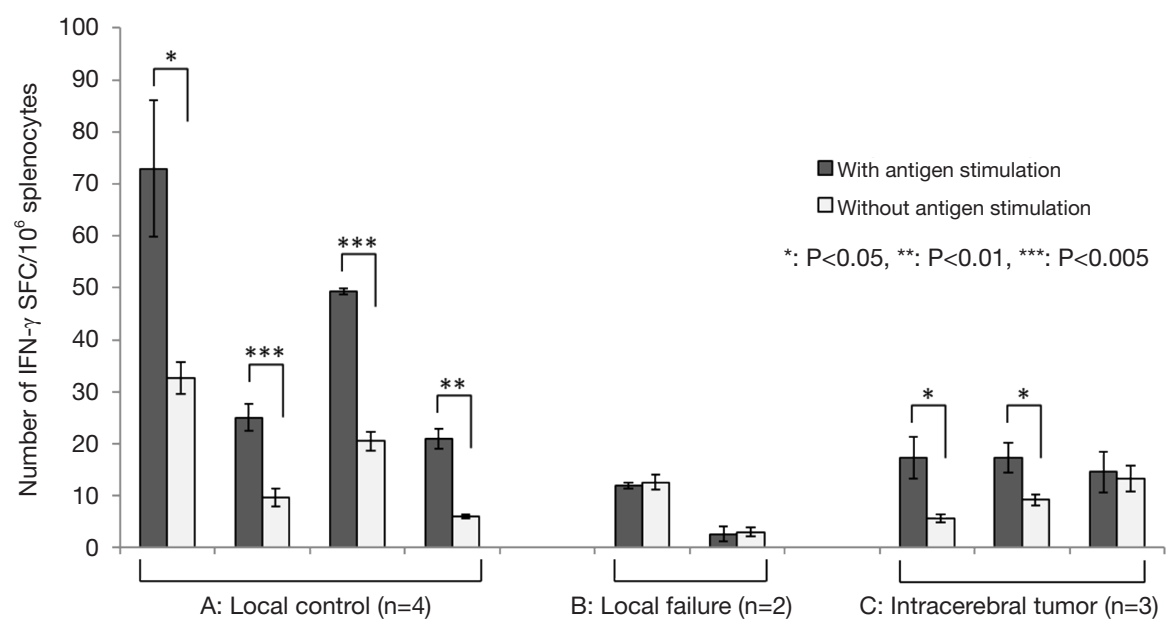

Figure 8 Results of ELISPOT assay. Black bars indicate the number of spots after antigen stimulation, and white bars indicate the number of spots without antigen stimulation. (A) The mice pre-treated by in situ-vaccination with local control (n=4); (B) the mice pre-treated by in situ-vaccination with local failure $(\mathrm{n}=2)$; (C) the control mice with intracerebral tumors $(\mathrm{n}=3)$. 
syngeneic mouse glioma model, in which a variety of immunotherapies have been investigated to date. GL261 tumor cells reportedly have mutations in the $p 53$ and $\mathrm{K}$-ras oncogenes and express a low but detectable level of major histocompatibility complex (MHC) class I molecules $(16,17)$, indicating that the cells are moderately immunogenic (16). In contrast, our GL261 cells demonstrated a high level of MHC class I expression, with a positivity ratio of over $90 \%$ (data not shown). Upon reviewing the literature reporting similar experiments, Szatmári et al. reported mean survival times of between $25-55$ days, corresponding to the number of inoculated cells ranging from $1 \times 10^{5}$ to $1 \times 10^{2}$, indicating that their survival times may be shorter than ours despite the fact that they administered fewer inoculated cells (16). Because of the different passage methods (in vivo vs. in vitro passage) or long-term in vitro culture, the intrinsic immunogenicity may be altered, which can be one of the reasons for the substantial differences in the biological features between research groups. To monitor the volume of tumors using the in vivo imaging system, GL261 cells were transfected with a plasmid encoding the gene for monomeric Kusabira Orange protein (pCAG-hu2 mKO). The growth viability and radiosensitivity of GL261-mKO cells were identical to those of the native GL261 cells as observed in our previous experiments. Szatmári et al. reported a survival rate of approximately $40 \%$ with a 2 Gy irradiation, which is comparable to our results (16).

\section{The in situ-irradiated tumor cells induce a protective abscopal effect if the mice are cured}

Of the 16 mice that received the in situ vaccination, 8 exhibited local tumor control following irradiation, and all mice rejected the same tumor cells challenged in the brain. In contrast, the other 8 mice that exhibited local failure following irradiation fell into an anergy state, and the tumor cells that were inoculated into the brain were engrafted and grew rapidly without exception (Figures 2,3B). In the survival analysis on the total 16 mice, the early loss is reflected by the 8 locally-failed mice with anergy state, and the late plateau part is reflected by the 8 locallycured mice (Figure $3 A$ ). Furthermore, the survival of the 8 locally-failed mice was significantly worse than that of the 12 control mice bearing intracranial tumors (Figure 3B). We consider that the tumor cells inoculated in the brain of none-vaccinated mice were attacked by the innate immune system first showing a temporary shrinkage of tumors, and survived tumor cells grew exponentially afterwards. In contrast, in locally-failed mice, not only the tumor specific immunity but also the innate immunity was probably disturbed by immune inhibiting factors released from recurrent tumors cells resulting in rapid growth of tumors. Also, this phenomenon in the locally-failed mice may be similar to a "phoenix rising" of the tumor, which is defined as an unexpected rapid regrowth of surviving tumor cells following irradiation $(20,21)$. This phenomenon is reportedly induced by PGE2 produced by irradiated tumor cells, leading to rapid tumor regeneration (20).

Our results indicate that once the balance between local control and failure shifted to the control side, the mice successfully acquired systemic antitumor immunity. In contrast, when the balance tilted to the local failure side, the immune system fell into a state of anergy, and the immune response was suppressed. Although it is difficult to determine the specific factors associated with the mice that are locally cured or failed, the balance between the local control and failure may be dependent on the innate immune status of each host. Thus, an improvement of the peripheral tumor control rate may be associated with an increased rate of establishing systemic anti-tumor immunity. For this purpose, the concomitant use of local immune-stimulants (e.g., the local administration of cytokines or adjuvants), and radiation dose escalation within a safe range may be worth trying.

A therapeutic model is more meaningful than a protective model for the analysis of immunotherapy. We have previously tested a protocol in which tumor cells were simultaneously inoculated into the subcutaneous tissue and into the brain, and only the subcutaneous tumors were irradiated; however, the abscopal effect on the tumor in the brain was not clearly noted (data not shown). In our opinion, it may be difficult to regress already engrafted brain tumor cells using only a distant abscopal effect induced by localized irradiation in this model. Moreover, this may be due to the insufficient immunogenicity of GL261. To further enhance the abscopal effect, research on combinations of different types of immunotherapy may be warranted. Recently, a combination of localized irradiation and the administration of an immune checkpoint inhibitor, a cytotoxic T lymphocyte antigen-4 (CTLA-4) antibody, has been reported to provide favorable results $(22,23)$. Additionally, the enhancement of GL261 tumor cell immunogenicity has been attempted by using $\alpha$-GalCer as an adjuvant (18) or via the transfection of cytokineproducing genes to G1261 cells $(24,25)$ with favorable results. 


\section{Effect of the ex vivo-irradiated tumor cell vaccine}

The ex vivo X-ray-irradiated GL261-mKO cells acted as an effective tumor vaccine in this protective study. This is comparable to the results reported by Szatmári et al., in which an irradiated GL261 tumor cell vaccination was effective when administered 3 or 7 days before intracranial tumor transplantation. However, there was no significant effect when the vaccination was performed on the same day or 3 days following the intracranial transplantation (16).

It should be noted that a transfection of Kusabira Orange protein may alter the immunogenicity of the tumor cells used in our study. Rosenzweig et al. reported enhanced green fluorescent protein (eGFP)-induced MHC-restricted CTL responses mediated by CD8+ lymphocytes in rhesus macaques, and the eGFP-specific CTLs were able to lyse autologous CD34+ cells expressing eGFP (26). Although the immunogenicity of Kusabira Orange protein has not yet been clearly elucidated, we consider that the most of the immune response was induced by GL261 tumor antigens, since we confirmed that INF- $\gamma$ production levels after stimulation with original GL261 and with GL261-mKO were identical by ELISPOT assay using splenocytes of cured mice (data not shown).

When comparing in situ and ex vivo irradiation for vaccination, in situ vaccination induces immunogenic tumor cell death with release of danger signals, which triggers a sequential systemic immunological response. In contrast, vaccination with ex vivo irradiated cells may be less effective because of less inflammation resulting in a lower level of danger signals released at the local site. Also, from a clinical point of view, surgery to remove tumor tissue is required for preparing whole tumor cell vaccine by irradiation. In contrast, surgical tumor removal is not necessary for in situ vaccination, thereby, it may be indicated for a wider range of tumors compared to vaccination with ex vivo.

\section{Tumor re-challenge in the long-term surviving mice}

Here, we demonstrated that immunological memory was established in the peripherally cured mice after irradiation. The re-challenged GL261-mKO cells were completely rejected in these mice. In contrast, the implanted B16/ BL6 melanoma cells grew rapidly and killed the mice with a median survival time of 15 days. In our preliminary experiments, the median survival time of the mice that were initially inoculated with the same B16/BL6 melanoma cells was 12 days. In addition, the survival time of these initially- inoculated mice was significantly reduced compared to that of the mice with in situ vaccination (data not shown). It has been reported that the melanoma-associated antigens gp100 and trp2 are shared with glioma cells (27). Thus, the CTLs induced by these common antigens may cause a growth delay of melanoma cells in in situ vaccinated mice. However, since there is a vast majority of different antigens, the inoculated B16/BL6 cells eventually survived and regrew.

\section{Immune cell infiltration in the brain of tumor-bearing mice and ELISPOT assay}

We demonstrated that the infiltration of CD8+ cells surrounding the tumor inoculation site was observed in the cured mice (Figure 6). Biollaz et al. reported that different immune cell infiltration patterns occurred in intracranial and peripheral glioma cells inoculated in a murine model (28). They observed an infiltration of CD4+ and CD8+ T cells in intracerebral tumors nine days following inoculation (28). Therefore, the temporal reduction in the inoculated tumor cells immediately after implantation may be caused by an innate immune response of non-specific immune cells (e.g., microglia). Nimmerjahn et al. and Mariani et al. reported that substantial numbers of activated microglia surrounding the inoculated tumors were noted by 7 th day of inoculation $(29,30)$. Those findings may indicate that surveying microglial cells initially respond as innate immune cells against tumor cell implantation, and subsequently, CD8+ T cells function to eliminate the inoculated tumor cells. A further analysis is necessary to clarify the interactions and roles of these two types, innate and acquired, immune cells.

In addition, using an ELISPOT assay with spleen cells, we showed that tumor antigen-specific IFN- $\gamma$ release was significantly greater in locally-cured mice than in the failed mice (Figure 8). This supports the fact that mice with local control after the in situ vaccination acquired tumor-specific anti-tumor immunity.

\section{Conclusions}

In conclusion, the in situ-irradiated tumor cell vaccine was effective in this protective protocol using the GL261 brain tumor model. In particular, X-ray irradiation to the peripheral subcutaneous tumors evoked a tumor-specific immune response (i.e., the protective abscopal effect) in the brain if the subcutaneous tumors were successfully cured. 
This may indicate that the systemic anti-tumor immune balance largely depends on local tumor control following irradiation.

\section{Acknowledgements}

We are indebted to Ms. Yoko Mori for her competent technical assistance.

Funding: This work was supported in part by JSPS KAKENHI Grant Number JP16K10384.

\section{Footnote}

Conflict of Interest: Tadao Ohno is the CEO and a stock holder of Cell-Medicine, Inc.

Ethical statement: All animal experimental procedures were conducted in accordance with a protocol approved by the Animal Experimentation Committee of the Faculty of Medicine, University of Tsukuba (16-386).

\section{References}

1. Formenti SC, Demaria S. Radiation therapy to convert the tumor into an in situ vaccine. Int J Radiat Oncol Biol Phys 2012;84:879-80.

2. Demaria S, Bhardwaj N, McBride WH, et al. Combining radiotherapy and immunotherapy: a revived partnership. Int J Radiat Oncol Biol Phys 2005;63:655-66.

3. Frey B, Rubner Y, Kulzer L, et al. Antitumor immune responses induced by ionizing irradiation and further immune stimulation. Cancer Immunol Immunother 2014;63:29-36.

4. Postow MA, Callahan MK, Barker CA, et al. Immunologic correlates of the abscopal effect in a patient with melanoma. N Engl J Med 2012;366:925-31.

5. Krysko DV, Garg AD, Kaczmarek A, et al. Immunogenic cell death and DAMPs in cancer therapy. Nat Rev Cancer 2012;12:860-75

6. Garg AD, Krysko DV, Verfaillie T, et al. A novel pathway combining calreticulin exposure and ATP secretion in immunogenic cancer cell death. EMBO J 2012;31:1062-79.

7. McBride WH, Chiang CS, Olson JL, et al. A sense of danger from radiation. Radiat Res 2004;162:1-19.

8. Obeid M, Tesniere A, Ghiringhelli F, et al. Calreticulin exposure dictates the immunogenicity of cancer cell death. Nat Med 2007;13:54-61.

9. Krysko O, Løve Aaes T, Bachert C, et al. Many faces of
DAMPs in cancer therapy. Cell Death Dis 2013;16;4:e631.

10. Reynders K, Illidge T, Siva S, et al. The abscopal effect of local radiotherapy: using immunotherapy to make a rare event clinically relevant. Cancer Treat Rev 2015;41:503-10.

11. Abuodeh Y, Venkat P, Kim S. Systematic review of case reports on the abscopal effect. Curr Probl Cancer 2016;40:25-37.

12. Ishiyama H, Teh BS, Ren H, et al. Spontaneous regression of thoracic metastases while progression of brain metastases after stereotactic radiosurgery and stereotactic body radiotherapy for metastatic renal cell carcinoma: abscopal effect prevented by the blood-brain barrier? Clin Genitourin Cancer 2012;10:196-8.

13. Stamell EF, Wolchok JD, Gnjatic S, et al. The abscopal effect associated with a systemic anti-melanoma immune response. Int J Radiat Oncol Biol Phys 2013;85:293-5.

14. Ransohoff RM, Brown MA. Innate immunity in the central nervous system. J Clin Invest 2012;122:1164-71.

15. Yang I, Han SJ, Kaur G, et al. The role of microglia in central nervous system immunity and glioma immunology. J Clin Neurosci 2010;17:6-10.

16. Szatmári T, Lumniczky K, Désaknai S, et al. Detailed characterization of the mouse glioma 261 tumor model for experimental glioblastoma therapy. Cancer Sci 2006;97:546-53.

17. Maes W, Van Gool SW. Experimental immunotherapy for malignant glioma: lessons from two decades of research in the GL261 model. Cancer Immunol Immunother 2011;60:153-60.

18. Hunn MK, Farrand KJ, Broadley KW, et al. Vaccination with irradiated tumor cells pulsed with an adjuvant that stimulates NKT cells is an effective treatment for glioma. Clin Cancer Res 2012;18:6446-59.

19. National Institutes of Health, Animal Research Advisory Committee. NIH Guidelines for Endpoints in Animal Study Proposals. (24 August 2016, date last accessed). Available online: https://oacu.oir.nih.gov/sites/default/ files/uploads/arac-guidelines/asp_endpoints.pdf

20. Li F, Huang Q, Chen J, et al. Apoptotic cells activate the "phoenix rising" pathway to promote wound healing and tissue regeneration. Sci Signal 2010;3:ra13.

21. Ng WL, Huang Q, Liu X, et al. Molecular mechanisms involved in tumor repopulation after radiotherapy. Transl Cancer Res 2013;2:442-8.

22. Yoshimoto Y, Suzuki Y, Mimura K, et al. Radiotherapyinduced anti-tumor immunity contributes to the therapeutic efficacy of irradiation and can be augmented by CTLA-4 blockade in a mouse model. PLoS One 
2014;31;9:e92572.

23. Belcaid Z, Phallen JA, Zeng J, et al. Focal radiation therapy combined with 4-1BB activation and CTLA-4 blockade yields long-term survival and a protective antigen-specific memory response in a murine glioma model. PLoS One 2014;9:e101764.

24. Lumniczky K, Desaknai S, Mangel L, et al. Local tumor irradiation augments the antitumor effect of cytokineproducing autologous cancer cell vaccines in a murine glioma model. Cancer Gene Ther 2002;9:44-52.

25. Newcomb EW, Demaria S, Lukyanov Y, et al. The combination of ionizing radiation and peripheral vaccination produces long-term survival of mice bearing established invasive GL261 gliomas. Clin Cancer Res 2006;12:4730-7.

26. Rosenzweig M, Connole M, Glickman R, et al. Induction of cytotoxic $\mathrm{T}$ lymphocyte and antibody responses

Cite this article as: Zenkoh J, Gerelchuluun A, Wang Y, Miwa Y, Ohno T, Tsuboi K. The abscopal effect induced by in situ-irradiated peripheral tumor cells in a murine GL261 brain tumor model. Transl Cancer Res 2017;6(1):136-148. doi: 10.21037/tcr.2017.01.32 to enhanced green fluorescent protein following transplantation of transduced CD34(+) hematopoietic cells. Blood 2001;97:1951-9.

27. Prins RM, Odesa SK, Li au LM. Immunotherapeutic targeting of shared melanoma-associated antigens in a murine glioma model. Cancer Res 2003;63:8487-91.

28. Biollaz G, Bernasconi L, Cretton C, et al. Site-specific anti-tumor immunity: differences in DC function, TGFbeta production and numbers of intratumoral Foxp3+ Treg. Eur J Immunol 2009;39:1323-33.

29. Nimmerjahn A, Kirchhoff F, Helmchen F. Resting microglial cells are highly dynamic surveillants of brain parenchyma in vivo. Science 2005;308:1314-8.

30. Mariani CL, Kouri JG, Streit WJ. Rejection of RG-2 gliomas is mediated by microglia and T lymphocytes. J Neurooncol 2006;79:243-53. 